\title{
Exploring extra dimensions to capture saliva metabolite fingerprints from metabolically healthy and unhealthy obese patients by comprehensive two-dimensional gas chromatography featuring Tandem lonization mass spectrometry
}

\author{
Marta Cialiè Rosso ${ }^{1} \cdot$ Federico Stilo $^{1} \cdot$ Simone Squara $^{1} \cdot$ Erica Liberto ${ }^{1} \cdot$ Stefania Mai $^{2} \cdot$ Chiara Mele $^{2,3}$. \\ Paolo Marzullo ${ }^{2,3}$. Gianluca Aimaretti ${ }^{3}$. Stephen E. Reichenbach ${ }^{4,5}$ - Massimo Collino ${ }^{1} \cdot$ Carlo Bicchi $^{1}$. \\ Chiara Cordero ${ }^{1}$
}

Received: 3 August 2020 / Revised: 1 October 2020 / Accepted: 13 October 2020 / Published online: 3 November 2020

(C) The Author(s) 2020

\begin{abstract}
This study examines the information potential of comprehensive two-dimensional gas chromatography combined with time-offlight mass spectrometry (GC $\times$ GC-TOF MS) and variable ionization energy (i.e., Tandem Ionization ${ }^{\mathrm{TM}}$ ) to study changes in saliva metabolic signatures from a small group of obese individuals. The study presents a proof of concept for an effective exploitation of the complementary nature of tandem ionization data. Samples are taken from two sub-populations of severely obese (BMI $>40 \mathrm{~kg} / \mathrm{m}^{2}$ ) patients, named metabolically healthy obese (MHO) and metabolically unhealthy obese (MUO). Untargeted fingerprinting, based on pattern recognition by template matching, is applied on single data streams and on fused data, obtained by combining raw signals from the two ionization energies (12 and $70 \mathrm{eV})$. Results indicate that at lower energy (i.e., $12 \mathrm{eV}$ ), the total signal intensity is one order of magnitude lower compared to the reference signal at $70 \mathrm{eV}$, but the ranges of variations for $2 \mathrm{D}$ peak responses is larger, extending the dynamic range. Fused data combine benefits from $70 \mathrm{eV}$ and $12 \mathrm{eV}$ resulting in more comprehensive coverage by sample fingerprints. Multivariate statistics, principal component analysis (PCA), and partial least squares discriminant analysis (PLS-DA) show quite good patient clustering, with total explained variance by the first two principal components (PCs) that increases from $54 \%$ at $70 \mathrm{eV}$ to $59 \%$ at $12 \mathrm{eV}$ and up to $71 \%$ for fused data. With PLSDA, discriminant components are highlighted and putatively identified by comparing retention data and $70 \mathrm{eV}$ spectral signatures. Within the most informative analytes, lactose is present in higher relative amount in saliva from MHO patients, whereas Nacetyl-D-glucosamine, urea, glucuronic acid $\gamma$-lactone, 2-deoxyribose, N-acetylneuraminic acid methyl ester, and 5aminovaleric acid are more abundant in MUO patients. Visual feature fingerprinting is combined with pattern recognition algorithms to highlight metabolite variations between composite per-class images obtained by combining raw data from individuals belonging to different classes, i.e., MUO vs. MHO.
\end{abstract}

Keywords Comprehensive two-dimensional gas chromatography-time of flight mass spectrometry · Variable ionization energy · Untargeted fingerprinting by template matching $\cdot$ Saliva metabolome $\cdot$ Fused data from multiplexed ionization

Marta Cialiè Rosso and Federico Stilo contributed equally to this work.

Electronic supplementary material The online version of this article (https://doi.org/10.1007/s00216-020-03008-6) contains supplementary material, which is available to authorized users.

Paolo Marzullo

paolo.marzullo@med.uniupo.it

Chiara Cordero

chiara.cordero@unito.it

1 Dipartimento di Scienza e Tecnologia del Farmaco, Università degli Studi di Torino, Via Pietro Giuria 9, 10125 Torino, Italy
2 Division of General Medicine, IRCCS Istituto Auxologico Italiano Ospedale S. Giuseppe, 28824 Piancavallo, Italy

3 Department of Translational Medicine, University of Piemonte Orientale, 28100 Novara, Italy

4 Computer Science and Engineering Department, University of Nebraska, Lincoln, NE 68588, USA

5 GC Image, LLC, Lincoln, NE 68508, USA 


\section{Introduction}

Comprehensive two-dimensional gas chromatography combined with time-of-flight mass spectrometric detection (GC $\times$ GC-TOF MS) is a multidimensional analytical platform with great potentials for profiling and fingerprinting of complex samples such as those typically considered in metabolomics studies (e.g., bio-fluids, tissue extracts, and exhaled volatile organic compounds [VOCs]) [1-7]. By this technique, known and unknown analytes (i.e., targeted and untargeted compounds) can be profiled in detail (i.e., detailed profiling) thanks to the great separation power and the high sensitivity achieved by band focusing with thermal modulation [8-11]. Moreover, a new concept of informative fingerprinting can be exploited through the investigation of 2D separation patterns with dedicated algorithms $[4,12]$. In this case, chromatographic fingerprinting and pattern recognition provide effective cross-sample analysis and sample-class discrimination, with the intrinsic potential of also using analyte metadata for identification. By this technique, untargeted investigations can be followed by post-targeting to achieve a higher level of information, helping in the interpretation of complex biological phenomena. Human obesity is a particularly suitable clinical condition owing to its multifold association with adverse cardiometabolic events [13].

Mass spectrometry (MS) is a fundamental dimension for any $\mathrm{GC} \times \mathrm{GC}$ platform, especially for complex samples analysis. MS adds another dimension to the system, providing orthogonal information that can be exploited to make fingerprinting more specific, for example by template matching with MS similarity constraints [14], and more accurate when quantitation is performed.

Variable electron ionization (EI) MS systems can increase the dimensionality of the analytical system and thus the specificity of the comparative exploration. Lowering the ionization energy can be done by simply setting the system to operate in such a condition [15] or by patented technologies that enable acquisition by time-switching between two ionization energies. The latter, termed Tandem Ionisation ${ }^{\mathrm{TM}}$ [Select-eVTM - US patent number 9,786,480], operates with variable-energy EI across single analytical runs. This patented system adopts, in the ion source, high potentials to accelerate electrons while reducing their energy through the ion chamber [16]. This results in more efficient ionization and reduced loss of sensitivity at low-energy EI with enhanced intensity for structure-diagnostic ions [17].

Recent applications of this technique in the field of motor oils [18] and diesel [19], blood volatiles [20], and food [17, 21] demonstrated that the combination of low ionization energies $(10-16 \mathrm{eV})$ with standard $70 \mathrm{eV}$ provide successful discrimination of samples, with open possibilities to adopt dedicated tandem data processing procedures.
In this study, variable ionization energy TOF MS is explored, for the first time, as an additional dimension of a $\mathrm{GC} \times \mathrm{GC}$ platform in saliva metabolome profiling and fingerprinting. In particular, the differential performance of an untargeted fingerprinting algorithm, based on template matching, is examined by processing single (separate low and high ionization energy) data streams and fused (sum of low and high ionization energy) data streams. The information potential of the system is evaluated through its sensitivity (absolute and relative), response dynamic range, signal response correlation, and fingerprint feature coverage. The challenges provided by this specific application are rooted in the complex metabolic derangement induced by obesity, further complicated by comorbidities occurring in a subset of recruited individuals.

For noninvasive diagnosis and monitoring of local and general diseases, saliva is very accessible and easy to collect. Unlike blood collection, which is almost always uncomfortable and painful for the patient, or feces or urine analysis, which makes people feel embarrassed, saliva collection is highly accessible, by a simple spit into sterile receptacles. Repeated samples can be collected, and diagnosis and monitoring using this method is an innovative and attractive approach [22].

Most notably, saliva metabolites have been noted to parallel metabolic alterations that occur in blood and may thus reflect many pathophysiological and nutritional changes, as well as exposure to medication and environmental factors [23]. Here, we propose to test saliva as a reliable bio-fluid to reveal informative metabolite patterns to determine whether there is a specific salivary signature of obesity per se. There is a limited number of reports on saliva of obese people. Obesity has been demonstrated to lead to a change in the salivary concentration of free sialic acid, total protein, and phosphate as well as activity of peroxidase, which contributes to the formation of dental caries [24]. While previous investigations revealed a reduced antioxidant status and increased proinflammatory cytokine expression in obese individuals, other salivary mediators relevant to obesity could be of metabolic importance for potential clinical and therapeutic implications $[25,26]$. However, the majority of obese patients suffer from comorbidities that may modulate saliva secretion either directly or through a required treatment, thus making it difficult to distinguish which changes in salivary parameters could be attributed purely to an increase in body mass. For these reasons, here we focused our analysis on two different populations of subjects with severe obesity (body mass index, BMI $\geq 40 \mathrm{~kg} / \mathrm{m}^{2}$ ), according to their metabolic status. Specifically, the recruited subjects were defined as "metabolically unhealthy" obese (MUO) or "metabolically healthy" obese (MHO), depending on whether or not they followed normal metabolic parameters. Generally speaking, a person with MHO has a BMI high enough to be classified as obese but is without the metabolic abnormalities that are usually linked to obesity [27]. 


\section{Materials and methods}

\section{Reference compounds and solvents}

Pure standards of $n$-alkanes (from $n-\mathrm{C}_{7}$ to $n$ - $\mathrm{C}_{30}$ ) for linear retention index $\left(I^{T}\right)$ determination and 1,4-dibromobenzene and 4-fluorophenylalanine for system evaluation and internal standardization were from Merck (Milan, Italy).

The $n$-alkanes mixture was prepared in cyclohexane at a concentration of $100 \mathrm{mg} \mathrm{L}^{-1}$; the internal standard (IS) 1,4dibromobenzene solution was prepared in toluene at a concentration of $10 \mathrm{~g} \mathrm{~L}^{-1}$ and spiked to ready-to-inject derivatized saliva samples at $50 \mathrm{mg} \mathrm{L}^{-1}$. The process verification IS, 4fluorophenylalanine, was prepared in methanol at a concentration of $10 \mathrm{~g} \mathrm{~L}^{-1}$ and spiked to saliva aliquots before the derivatization process at a final concentration of $50 \mathrm{mg} \mathrm{L}^{-1}$.

Pure standards for identity confirmation of pyruvic acid, lactic acid, malonic acid, acetoacetic acid, phosphoric acid, succinic acid, glyceric acid, fumaric acid, malic acid, citric acid, alanine (Ala), asparagine (Asn), aspartic acid (Asp), cysteine (Cys), glutamic acid (Glu), glycine (Gln), isoleucine (Ile), leucine (Leu), lysine (Lys), methionine (Met), ornithine (Orn), phenylalanine (Phe), proline (Pro), serine (Ser), threonine (Thr), tryptophan (Trp), tyrosine (Tyr), valine (Val), glycerol, xylitol, mannitol, myo-inositol, fructose, galactose, glucose, saccharose, lactose, and 4-chlorophenylalanine (internal quality control [IQC] for derivatization) were from Merck (Milan, Italy).

Derivatization reagents and $\mathrm{HPLC}$-grade solvents $\mathrm{O}$ methylhydroxylamine hydro-chloride (MOX), N,Obis(trimethylsilyl)trifluoroacetamide (BSTFA), methanol, pyridine, $n$-hexane, dichloromethane, and toluene were from Merck (Milan, Italy).

\section{Saliva samples and quality controls}

This study includes patients recruited at the Istituto Auxologico Italiano, Verbania, Italy [28, 29]. The height, weight, and waist circumference of participants were measured as a part of regular assessment. Other subjects with body mass index (BMI) of $40 \mathrm{~kg} / \mathrm{m}^{2}$ or greater were recruited for the study. In total, 34 obese men (BMI $\left.\geq 40 \mathrm{~kg} / \mathrm{m}^{2}\right)$ were then defined as metabolically unhealthy (MUO, $n=24$ ) or metabolically healthy (MHO, $n=10$ ), depending on whether their metabolic parameters were within normal ranges, i.e. high fasting triglycerides, $1.7 \mathrm{mmol} / \mathrm{L}$ or greater $(\geq 150 \mathrm{mg} / \mathrm{dL})$; reduced HDL cholesterol level $(<1.03 \mathrm{mmol} / \mathrm{L}[<40 \mathrm{mg} /$ $\mathrm{dL}]$ ); and whether their blood pressure was elevated $(\geq 130 \mathrm{mmHg}$ systolic blood pressure or $\geq 85 \mathrm{mmHg}$ diastolic blood pressure) or on antihypertensive drug treatment and fasting glucose concentration $\geq 5.6 \mathrm{mmol} / \mathrm{L}(\geq 100 \mathrm{mg} / \mathrm{dL})$. These criteria were taken from the metabolic syndrome definition [30] and have been used in similar studies. The experimental procedure was approved by the ad hoc Ethical Research Committee of the Istituto Auxologico Italiano (Verbania, Italy). Written informed consent was obtained from the patients. The study protocol conformed to the guidelines of the European Convention on Human Rights and Biomedicine concerning biomedical research.

Saliva from a subset of obese individuals (MHO $n=3$ and MUO $n=5$ ) randomly selected over the available samples and quality controls (QCs) obtained by healthy, normal-weight males $(n=4)$ of the same age were subjected to a standard derivatization protocol [31] adjusted to comply for method sensitivity and metabolite coverage. Sample preparation consisted of the following steps: $100 \mu \mathrm{L}$ of saliva and a suitable volume of process IS (4-fluorophenylalanine) were carefully mixed (Fisherbrand Whirlimixer vortex; Fisher Scientific, Loughborough, Leicestershire, UK). Then, the solution was dried under a gentle stream of nitrogen before the addition of $25 \mu \mathrm{L}$ of $\mathrm{MOX}(20 \mathrm{mg} / \mathrm{mL}$ in pyridine) and left to react for $2 \mathrm{~h}$ at $60^{\circ} \mathrm{C}$. Next, $75 \mu \mathrm{L}$ of BSTFA were added, and the mixture was incubated at $60{ }^{\circ} \mathrm{C}$ for $1 \mathrm{~h}$. The resulting sample solution was spiked with 1,4-dibromobenzene to a final concentration of $50 \mathrm{mg} \mathrm{L}^{-1}$ and diluted in toluene to a final volume of $200 \mu \mathrm{L}$. Analyses were immediately run with duplicate injections for each sample; samples were randomized within a $24 \mathrm{~h}$ time frame after derivatization.

\section{GCXGC-TOF MS and Tandem Ionization ${ }^{\mathrm{TM}}$ : Instrument set-up and conditions}

GC $\times$ GC analyses were performed on an Agilent 7890B GC unit coupled with a Bench TOF-Select ${ }^{\mathrm{TM}}$ system (Markes International, Llantrisant, UK) featuring tandem EI equipped with a SepSolve (Llantrisant, UK) preparation robot with automated tool change model PAL3-RTC. Hard ionization at $70 \mathrm{eV}$ was set for identity confirmation, while $12 \mathrm{eV}$ was applied to explore spectral and response complementarity. The ion source and transfer line were set at $290^{\circ} \mathrm{C}$. The MS optimization option was set to operate in Tandem Ionisation ${ }^{\mathrm{TM}}$ with a mass range between 45 and $1000 \mathrm{~m} / \mathrm{z}$; data acquisition frequency was $50 \mathrm{~Hz}$ per channel; filament voltage was set at $1.60 \mathrm{~V}$.

The system was equipped with a two-stage KT 2004 loop thermal modulator (Zoex Corporation, Houston, TX, USA) cooled with liquid nitrogen controlled by an Optimode ${ }^{\mathrm{TM}}$ V.2 controller (SRA Instruments, Cernusco sul Naviglio, Milan, Italy). The hot-jet pulse time was set at $350 \mathrm{~ms}$; modulation period was $5 \mathrm{~s}$; and cold-jet total flow was progressively reduced with a linear function from $30 \%$ of the mass flow controller (MFC) at initial conditions to $8 \%$ at the end of the run.

The column set was configured as follows: ${ }^{1} \mathrm{D}$ DB-5 column $(95 \%$ polydimethylsiloxane, $5 \%$ phenyl; $30 \mathrm{~m} \times$ $0.25 \mathrm{~mm} \mathrm{~d}_{\mathrm{c}}, 0.25 \mu \mathrm{m} \mathrm{d}_{\mathrm{f}}$ ) coupled with a ${ }^{2} \mathrm{D}$ OV1701 column (86\% polydimethylsiloxane, $7 \%$ phenyl, $7 \%$ cyanopropyl; $2 \mathrm{~m} \times 0.1 \mathrm{~mm} \mathrm{~d}_{\mathbf{c}}, 0.10 \mu \mathrm{m} \mathrm{d}_{\mathbf{f}}$ ) from J\&W (Agilent, Little 
Falls, DE, USA). The first $0.80 \mathrm{~m}$ of the ${ }^{2} \mathrm{D}$ column, connected in series to the ${ }^{1} \mathrm{D}$ column by a silTite $\mu$-union (Trajan Scientific and Medical, Ringwood, Victoria, Australia), were wrapped in the modulator slit and used as a loop capillary for cryogenic modulation. The carrier gas was helium at a constant flow of $1.6 \mathrm{~mL} \mathrm{~min}{ }^{-1}$. The oven temperature program was from $70{ }^{\circ} \mathrm{C}(2 \mathrm{~min})$ to $120{ }^{\circ} \mathrm{C}$ at $10{ }^{\circ} \mathrm{C} \mathrm{min}{ }^{-1}$, then to $320^{\circ} \mathrm{C}(1 \mathrm{~min})$ at $4{ }^{\circ} \mathrm{C} \mathrm{min}^{-1}$.

For saliva samples, $2.0 \mu \mathrm{L}$ of the derivatized solution ("Saliva samples and quality controls" section) were analyzed under the following conditions: split/splitless injector in split mode, split ratio $1: 20$, and injector temperature $300{ }^{\circ} \mathrm{C}$. The $n$-alkane liquid sample solution for $I^{T}$ determination was analyzed under the following conditions: split/splitless injector in split mode, split ratio $1: 50$, injector temperature $300^{\circ} \mathrm{C}$, and injection volume $1 \mu \mathrm{L}$.

\section{Raw data acquisition and GCXGC data processing}

Data were acquired by TOF-DS software (Markes International, Llantrisant, UK) and processed using GC Image $\mathrm{GC} \times \mathrm{GC}$ Software 2.9 (GC Image, LLC, Lincoln, NE, USA). Statistical analysis was performed by XLSTAT 2014 (Addinsoft, New York, NY, USA) and heat map visualization by GENE-E version 3.0.77 (Broad Institute, Inc. Cambridge, MA, USA).

\section{Method performance parameters: retention times and response repeatability}

Method validation was run for 2 weeks in order to evaluate the repeatability of retention times and untargeted and targeted (UT) peak response precision [32]. Retention times in both chromatographic dimensions $\left({ }^{1} t_{R}\right.$ and $\left.{ }^{2} t_{R}\right)$ were collected from the UT peak regions at $70 \mathrm{eV}$ of analyzed samples [ $(4 \mathrm{QCs}+8$ patients) $\times 2$ process replicates $\times 2$ analytical replicates] for a total of 48 analytical runs processed. Results are reported as $\%$ relative standard deviation (\% RSD) in Electronic Supplementary Material (ESM) Table S1, with quite good retention time stability, with an average RSD of $0.20 \%$ for ${ }^{1} t_{R}$ and $1.90 \%$ for ${ }^{2} t_{R}$. Response repeatability was calculated on 552 reliable peaks from fused data and was based on normalized responses from QC samples $(4 \mathrm{QCs} \times 2$ process replicates $\times 2$ analytical replicates $=16$ analytical runs) acquired over 2 weeks. As reported in ESM Table S1, repeatability \% RSD averaged 12.13, with a minimal value of 3.28 for peak feature \#134 and a maximum of 49.99 for \#31.

\section{UT fingerprinting workflow}

The 2D data elaboration workflow is illustrated in Fig. 1 and detailed in the ESM Fig. S1. It is based on an approach developed to comprehensively map UT features (i.e., peaks and peak regions) for the most inclusive fingerprinting [33, 34].
In this specific application, the pre-targeting of analytes was skipped by performing fully automated untargeted fingerprinting on parallel data streams generated by Tandem Ionization $^{\mathrm{TM}}$ (70 and $12 \mathrm{eV}$ channels). In addition, composite chromatograms $[17,19]$ were computed by summing the parallel 12 and $70 \mathrm{eV}$ data streams for each single analytical run, and submitted to the UT processing workflow.

The template-matching strategy [14] is at the basis of the comprehensive realignment of untargeted features across all chromatograms. The metadata corresponding to all detected 2D peaks and peak regions (retention times, MS fragmentation patterns, and detector responses) are actively used to establish reliable correspondences between chemical entities across multiple chromatograms. The specificity of the approach relies on the constraints applied to validate positive matches. Based on previous studies aimed at validating the robustness and the accuracy of the process [17, 35], a spectral-similarity threshold of 750 was imposed for both direct match factor (DMF) and reverse match factor (RMF) between reference (template) and analyzed (candidate) MS signatures using the NIST MS Search algorithm, version 2.0 (National Institute of Standards and Technology, Gaithersburg, MD, USA) [35]. Matching was between "peak spectra," i.e., the MS signature from the largest data point within the peak, and a signal-to-noise $(\mathrm{S} / \mathrm{N})$ threshold of 100 was fixed to discard 2D peaks with inconsistent MS information.

The output of the full untargeted fingerprinting is a data matrix of aligned 2D peaks and peak regions together with related metadata $\left({ }^{1} \mathrm{D}\right.$ and ${ }^{2} \mathrm{D}$ retention times, MS fragmentation pattern, base peak and molecular ion $\mathrm{m} / \mathrm{z}$, and total ion current [TIC] response), which is available for further processing [36-38]. Here, there are three independent data matrices, for the $70 \mathrm{eV}, 12 \mathrm{eV}$, and fused data streams.

The untargeted fingerprinting (Step 1 in Fig. 1) was performed automatically by GC Image Investigator ${ }^{\mathrm{TM}}$ version 2.9 software (GC Image, LLC, Lincoln, NE, USA) to generate peak and peak-region features [12, 39]. For this purpose, peak detection thresholds were set according to absolute responses (TIC counts) corresponding to $\mathrm{S} / \mathrm{N}$ threshold of 100 (i.e., 500,000 counts/ $70 \mathrm{eV} ; 5000$ counts/12 eV; 100,000 counts/fused data). Values were confirmed by visual supervision to yield comparable 2D peak detection across channels. The process $[4,12,33,38-40]$ aligned the 48 chromatograms [ $(4$ QCs +8 patients $) \times 2$ process replicates $\times 2$ analytical replicates] for each data stream $(12$ and $70 \mathrm{eV})$ plus the fused data $(12+70 \mathrm{eV})$ using a set of registration peaks, named reliable peaks, to produce a composite chromatogram from which peak regions were extracted. Reliable peaks are those 2D peaks that were positively matched across more than half of the chromatograms (i.e., 25 of the 48 sample chromatograms).

The resulting templates with untargeted (reliable) peaks and peak regions extracted by the composite chromatograms 


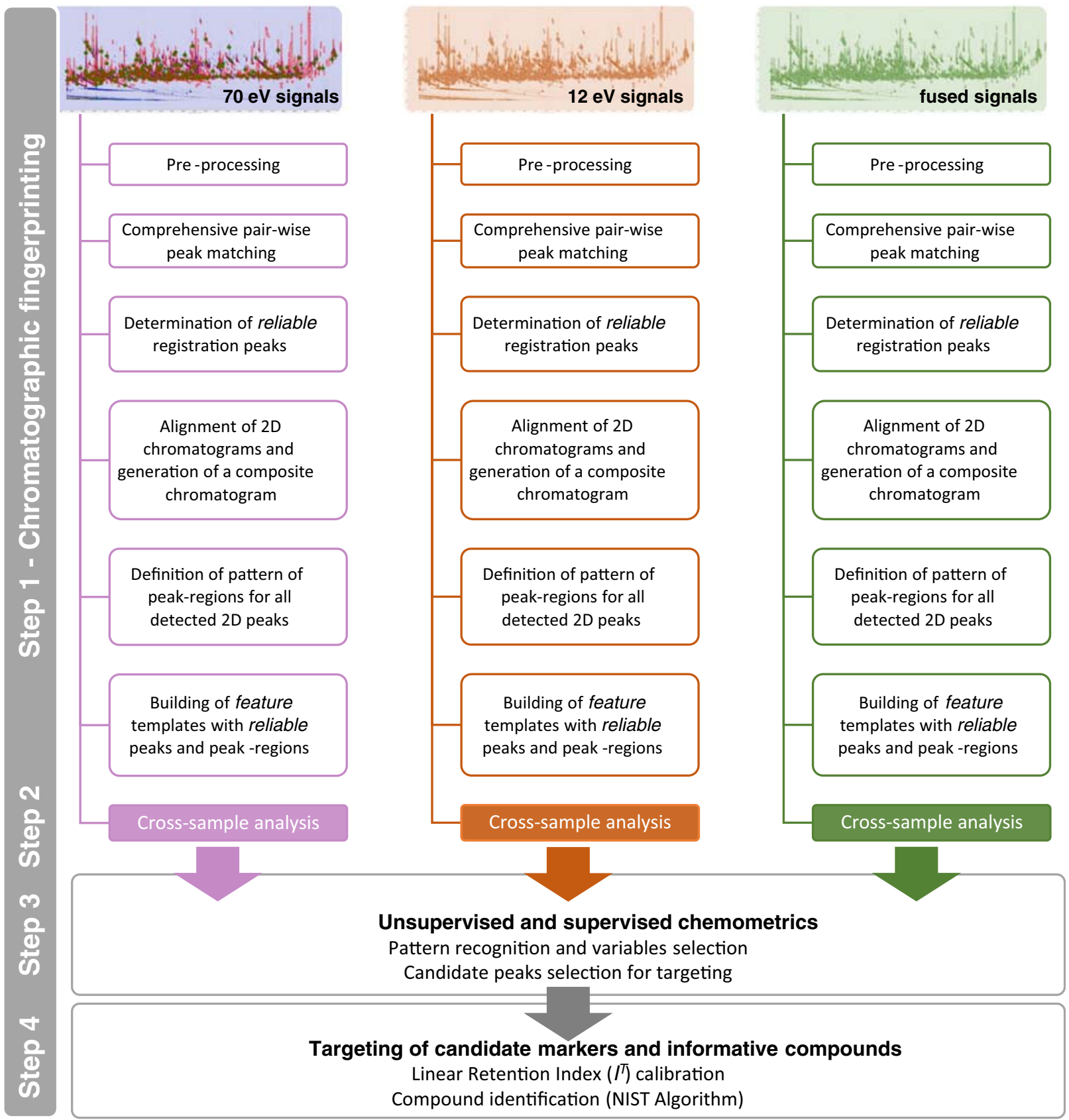

Fig. $12 \mathrm{D}$ data elaboration workflow based on untargeted fingerprinting on single $(12$ and $70 \mathrm{eV})$ and fused $(12+70 \mathrm{eV})$ data streams

(i.e., the feature templates) are shown in Fig. 2 where the saliva metabolome signature of an unhealthy obese (MUO) patient is shown as it results from the $70 \mathrm{eV}$ ionization channel (2a), $12 \mathrm{eV}(2 \mathrm{~b})$, and after fusion of the tandem data streams (2c). The visual complexity of the templates is indicative of the richness of the untargeted fingerprint. Details on the results of this processing are discussed in "Full untargeted fingerprinting on single and fused data streams" section.

Targeting of informative compounds and potential markers (Step 4 in Fig. 1) was performed for those peak regions that demonstrated higher information potential by unsupervised and supervised chemometrics (Step 3 in Fig. 1) for discriminating healthy from unhealthy obese saliva signatures. Identification of targeted analytes was carried out by matching candidate EI-MS fragmentation patterns at $70 \mathrm{eV}$ (NIST MS Search algorithm, version 2.0) with those collected in commercial and in-house databases (subject to a DMF threshold of 900 and RMF threshold of 950). In addition, linear retention indices $\left(I^{T}\right)$ were adopted as a further constraint; experimental values were compared with NIST reference indices using a tolerance of \pm 10 units. 

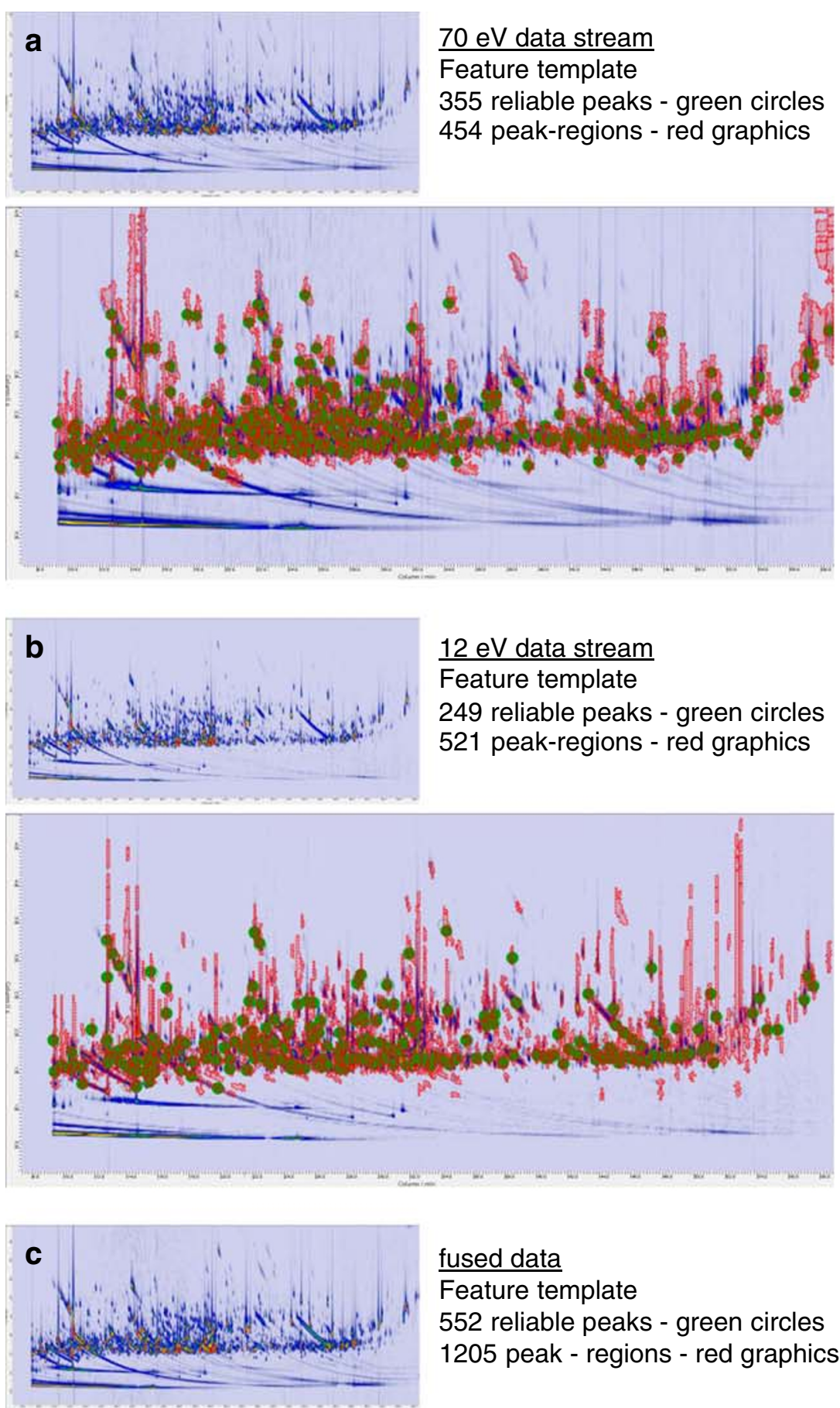

fused data

Feature template

552 reliable peaks - green circles

1205 peak - regions - red graphics

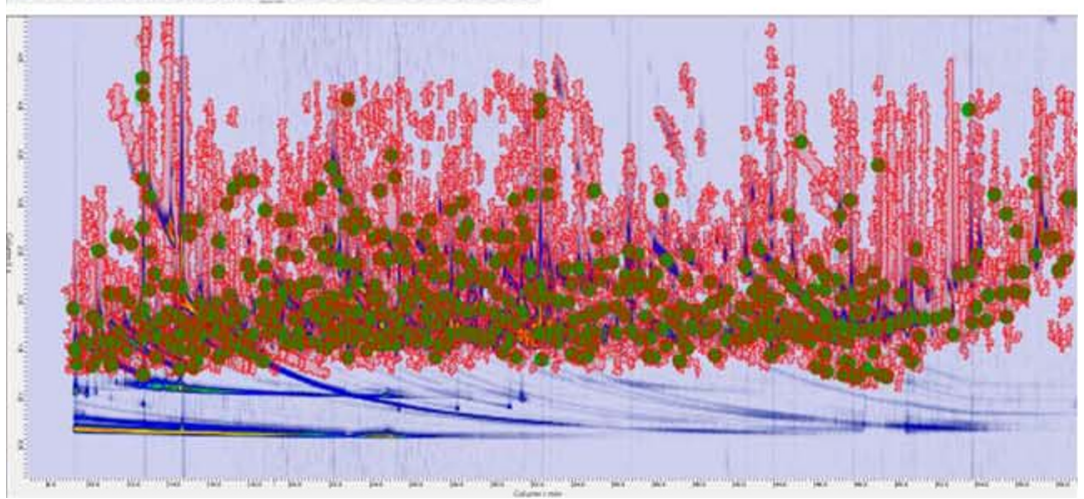


Fig. 2 Pseudo-color chromatographic images corresponding to an unhealthy obese (MUO) patient, resulting from the $70 \mathrm{eV}$ ionization channel (a), $12 \mathrm{eV}(\mathbf{b})$, and after fusion of the tandem data streams (c). Green circles indicate reliable 2D peaks, and red graphics indicate untargeted peak regions. Details on feature template objects are also reported

\section{Results and discussion}

In this study, the complex saliva metabolome was explored, for the first time, by combining the separation power of $\mathrm{GC} \times \mathrm{GC}$ with the complementary information provided by TOF MS with variable ionization energy. The fingerprinting process, based on pattern recognition (i.e., UT fingerprinting), was applied in its full flexibility to cross-align features (2D peaks and peak regions) between tandem signals and with the fused data, to validate the role of informative analytes and to facilitate their identification using the $70 \mathrm{eV}$ spectra.

The next sections discuss full untargeted fingerprinting results and performance obtained by independent processing of single $(70$ and $12 \mathrm{eV})$ and fused $(70 \mathrm{eV}+12 \mathrm{eV})$ data streams as illustrated at Steps 1 and 2 in Fig. 1).

\section{Full untargeted fingerprinting on single and fused data streams}

The sample set under consideration in this study exhibited several analytical challenges related to both the interindividual variability of the saliva metabolome [41] and its intrinsic complexity due to the concurrent presence of comorbidity and metabolic derangements in obese patients. In such a context, the combination of two ionization energies was beneficial due to their different and complementary dynamic ranges of response [19-21, 42]. It was demonstrated by previous investigations that when operating at lower ionization energies (e.g., 10-16 eV), the generalized reduction in analyte absolute response was accompanied by higher relative sensitivity, expressed as signal-to-noise ratio $(\mathrm{S} / \mathrm{N})$, for several analytes $[17,21]$. This higher relative sensitivity was much more evident for those analytes that showed the most dissimilar fragmentation patterns between tandem channels.

These benefits were exploited to delineate metabolite signatures capable of discriminating healthy from unhealthy obese patients. Of course, this preliminary study is just a proof of concept useful for evaluating the potential and the synergism of adding extra dimensions at the MS detection level in challenging scenarios. The limited number of samples available does not allow definitive generalized conclusions regarding metabolomics; however, the fine-tuning of 2D data processing strategies and the critical evaluation of the actual performance of different workflows will inform future investigations with larger sample sets.
The 2D data processing strategy was conducted in a full untargeted fashion, at the first stage. For a better understanding of the signal characteristics, here follows some details on: (a) background signal intensity before and after noise subtraction [43]; (b) number of detected 2D peaks above a fixed threshold; and (c) range of variation for $\mathrm{S} / \mathrm{N}$ and $2 \mathrm{D}$ peak absolute response. All these data are summarized in Table 1 and refer to average values computed from the MUO \#20 patient saliva replicates $(n=4)$.

Results confirm that at lower ionization energies, the total signal intensity is lower ( 1840 vs. 23,090 counts) by one order of magnitude, while for the fused data stream, the average background level is close to that at $70 \mathrm{eV}$ (26,140 vs. 23,090 counts). After noise subtraction, applied here according to the Sternberg algorithm [44], the resulting baseline was much more effectively reduced at $12 \mathrm{eV}$, where the reduction was of two orders of magnitude. This operation did not affect spectral quality, as demonstrated by the number of reliable peaks detected (see below).

The number of detected 2D peaks above the fixed TIC response thresholds ("UT fingerprinting workflow" section) and $\mathrm{S} / \mathrm{N} \geq 100$ was comparable between $70 \mathrm{eV}$ and the fused data streams, with the latter exceeding the reference channel (i.e., $70 \mathrm{eV}$ ) by $6 \%$. On the other hand, as expected, at lower energies, 2D peaks were $36 \%$ lower than those at $70 \mathrm{eV}$. Absolute response values, calculated on the TIC channel, span three orders of magnitude following a log-normal distribution (data not shown), but with a wider range of variation calculated for the $12 \mathrm{eV}$ channel: \% relative standard deviation (RSD) of $319 \%$ vs. $174 \%$ at $70 \mathrm{eV}$. This signal characteristic is of great interest because it enables exploration of complex fractions with a wider dynamic range. Analyte response/ concentration variations can be more effectively captured, especially on the higher range, at higher intensity values, where at $70 \mathrm{eV}$ detector saturation may easily occur. It should be stressed that with band compression in space of thermal modulation, the analyte concentration gradient entering the ion source and then reaching the detector is higher than with conventional 1D GC-MS. The fused data stream, combining 70 and $12 \mathrm{eV}$ signal characteristics, span a wider range of responses compared to the $70 \mathrm{eV}$ with larger RSD.

The impact of signal characteristics can be appreciated by untargeted fingerprinting processing ("UT fingerprinting workflow" section). The single and summed (i.e., fused) data streams were independently processed by Image Investigator software (GC Image, LLC), applying the most relaxed conditions [39] for reliable peak selection. By this approach, untargeted 2D peaks that are matched (subject to retention time constraints and $750 \mathrm{MS}$ similarity) across at least half of the chromatograms of the set ( $>24$ of the 48 individual chromatograms) are recorded and adopted as alignment points for the construction of a composite image (ESM Fig. S2) for 
Table 1 Data processing parameters based on single (12 and $70 \mathrm{eV})$ and fused $(12+70 \mathrm{eV})$ data streams. Untargeted fingerprinting statistics and supervised chemometrics are detailed throughout the text

\begin{tabular}{|c|c|c|c|}
\hline \multirow[t]{2}{*}{ Parameter } & \multicolumn{3}{|c|}{ Detection channel } \\
\hline & $70 \mathrm{eV}$ & $12 \mathrm{eV}$ & Fused data \\
\hline \multicolumn{4}{|c|}{ Average background intensity (counts) (retention window ${ }^{1} \mathrm{D} 15.5-19.0 \mathrm{~min} /{ }^{2} \mathrm{D} 4.5-5.0 \mathrm{~s}$ ) } \\
\hline Before noise subtraction & 23,090 & 1840 & 26,140 \\
\hline After noise subtraction & 1150 & 10 & 1140 \\
\hline No. detected $2 \mathrm{D}$ peaks $\mathrm{S} / \mathrm{N} \geq 100$ & 517 & 336 & 549 \\
\hline \multicolumn{4}{|l|}{ Absolute response TIC (peak volume) } \\
\hline Min & $5.17 \mathrm{E}+05$ & $2.63 \mathrm{E}+04$ & $1.08 \mathrm{E}+05$ \\
\hline Max & $1.69 \mathrm{E}+08$ & $4.59 \mathrm{E}+07$ & $2.12 \mathrm{E}+08$ \\
\hline RSD & $174 \%$ & $319 \%$ & $198 \%$ \\
\hline \multicolumn{4}{|l|}{ Signal-to-noise ratio $(\mathrm{S} / \mathrm{N}) \mathrm{TIC}$} \\
\hline Min & 100 & 100 & 100 \\
\hline Max & 59,007 & 36,209 & 52,954 \\
\hline RSD & $251 \%$ & $206 \%$ & $226 \%$ \\
\hline \multicolumn{4}{|l|}{ Fingerprinting and statistics } \\
\hline \multicolumn{4}{|l|}{ Feature template } \\
\hline No. reliable peaks & 355 & 249 & 552 \\
\hline No. peak regions & 454 & 521 & 1205 \\
\hline$\%$ Matched peaks on cumulative chromatogram & $99 \%$ & $97.6 \%$ & $95.3 \%$ \\
\hline No. $2 \mathrm{D}$ peaks $\mathrm{S} / \mathrm{N} \geq 100$ & 1954 & 1729 & 2267 \\
\hline \multicolumn{4}{|l|}{ Supervised statistics } \\
\hline \multicolumn{4}{|c|}{ Kruskal-Wallis-Dunn's test and Bonferroni correction } \\
\hline Peak regions meaningful $p$ (alpha 0.05 ) & 100 & 110 & 135 \\
\hline
\end{tabular}

each channel set. Results are summarized in Table 1 and discussed in the following text.

According to signal characteristics, the number of reliable peaks at $70 \mathrm{eV}$ was higher than at $12 \mathrm{eV}$ (355 vs. 249); this result is in line with absolute response data and relative sensitivity ( $\mathrm{S} / \mathrm{N}$ range), confirming that at higher ionization energies, the number of 2D peaks with consistent spectral information is larger. For the fused data stream, the number of reliable peaks was $55 \%$ greater than for $70 \mathrm{eV}$, indicating the benefits of adding low-ionization spectral information to the processing. At lower energies, the fragmentation is limited, and fragments with larger $m / z$ values prevail [17, 21], with benefits for spectral interpretation [45]. Spectral dissimilarity and fragmentation pattern characteristics are discussed in more detail for analytes of interest in this application in "Informative metabolites and their response from tandem signals" section.

The benefits of data fusion also are reflected in the number of untargeted peak regions delineated over the composite chromatogram(s) obtained by realignment and summing of single or fused data images for the 48 runs. Peak regions correspond to all detected 2D peaks above the TIC response fixed thresholds (i.e., 500,000, $70 \mathrm{eV} ; 5000,12 \mathrm{eV} ; 100,000$, fused data). Single ionization channels had almost the same coverage by mapping 454 and 521 untargeted peak regions at 70 and $12 \mathrm{eV}$, respectively, whereas the fused data doubled the feature coverage, reaching 1205 peak regions. This result emphasizes the advantages of processing hybrid data, exploiting in full the potential benefits of the tandem channels.

Last but not least, the feature template created from fused data has a greater compositional coverage. For the single ionization channels, the reliable peaks, with 355 at $70 \mathrm{eV}$ or 249 at $12 \mathrm{eV}$, achieved a $99 \%$ and $98 \%$ matching rate on the corresponding composite chromatograms, but covered only $18 \%$ and $14 \%$ of the detected 2D peaks. For fused data, the 552 reliable peaks achieved a $95.3 \%$ matching rate and covered $24 \%$ of the detected peaks.

The next section discusses results for unsupervised and supervised statistical analyses of the saliva patterns resulting from untargeted fingerprinting for the different detection channels. 2D peaks and peak regions with meaningful variations are then examined to determine a putative identity and hypothesize an informational role.

\section{Chromatographic feature selection and results cross- validation}

As a first unsupervised exploration, principal component analysis (PCA) was conducted for all analyzed samples (48 runs) using \% response (based on TIC 2D volumes) for realigned $2 \mathrm{D}$ peak regions from the feature template. At this stage, QC samples were included to provide a first impression of the 
Fig. 3 Principal component analysis (PCA) including all analyzed samples (48 runs) and based on $\%$ response (TIC $2 \mathrm{D}$ volumes) of realigned 2D peak regions from the feature template: a $70 \mathrm{eV}, \mathbf{c} 12 \mathrm{eV}$, e fused data streams. QC samples are retained to show response consistency and to illustrate the statistical distance between the saliva of healthy subjects (QCs) and that of MUO/ $\mathrm{MHO}$ patients. Feature reduction by the Kruskal-Wallis test with post hoc correction by Dunn's test and Bonferroni correction results in PCA loading plots of $\mathbf{b}$ $(70 \mathrm{eV}), \mathbf{d}(12 \mathrm{eV})$, and $\mathbf{f}$ (fused data streams)
70 eV - 454 Peak-regions F1-F2: $56.26 \%$

70 eV - p > 0.05 F1-F2: $54.39 \%$

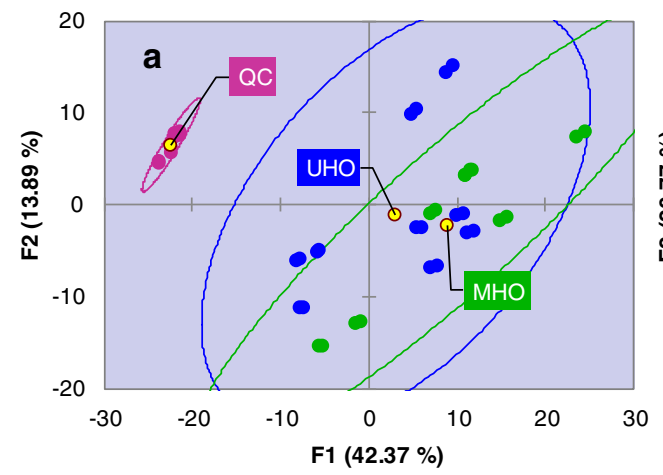

12 eV - 521 Peak-regions F1-F2: $52.27 \%$
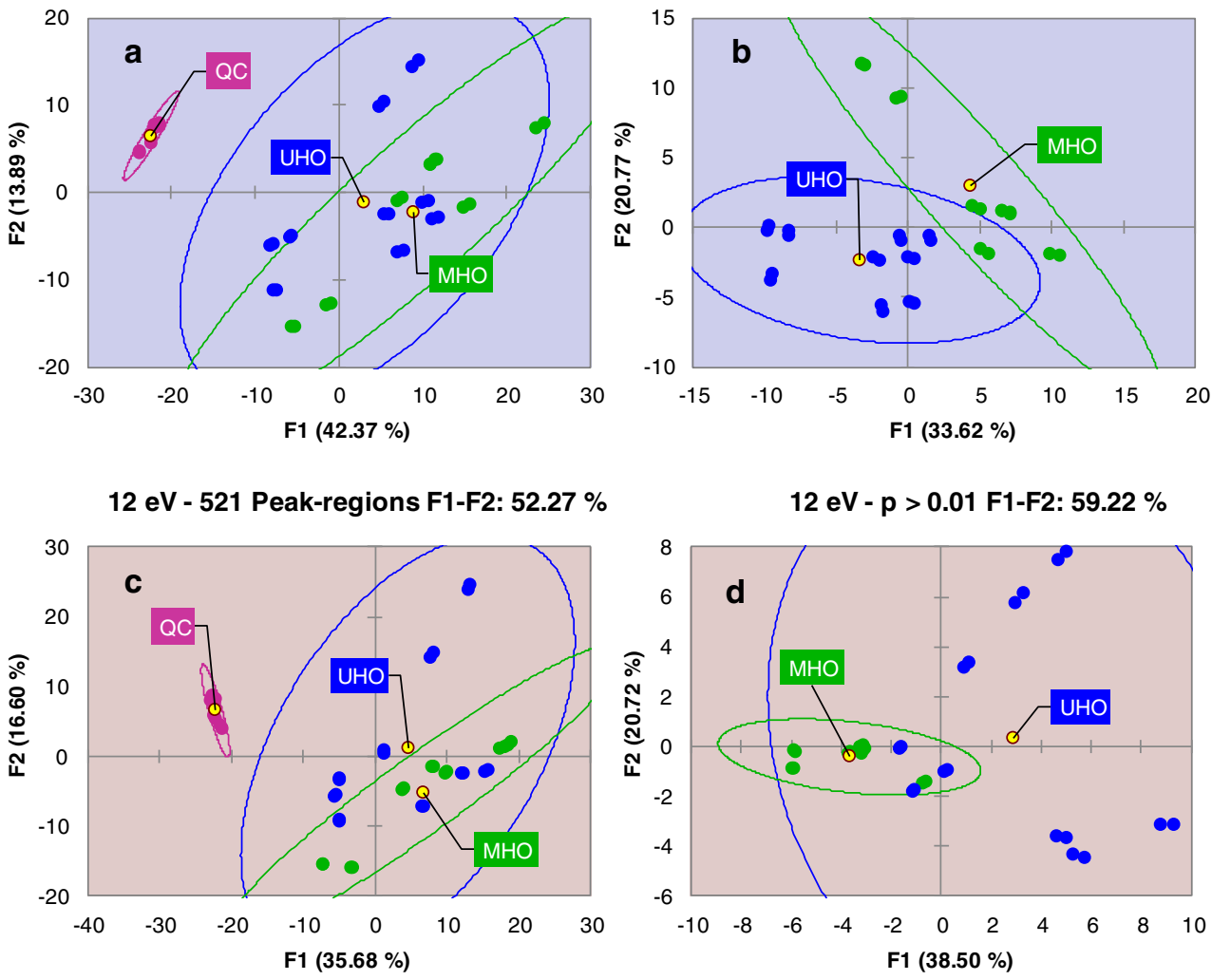

$12 \mathrm{eV}$ - p > 0.01 F1-F2: $59.22 \%$

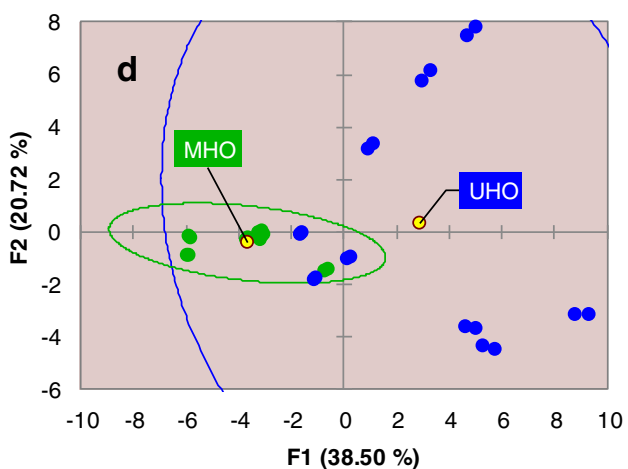

(12+70) eV - 1205 Peak-regions F1-F2: 50.24\%

(12+70) eV - p > 0.05 F1-F2: $71.40 \%)$
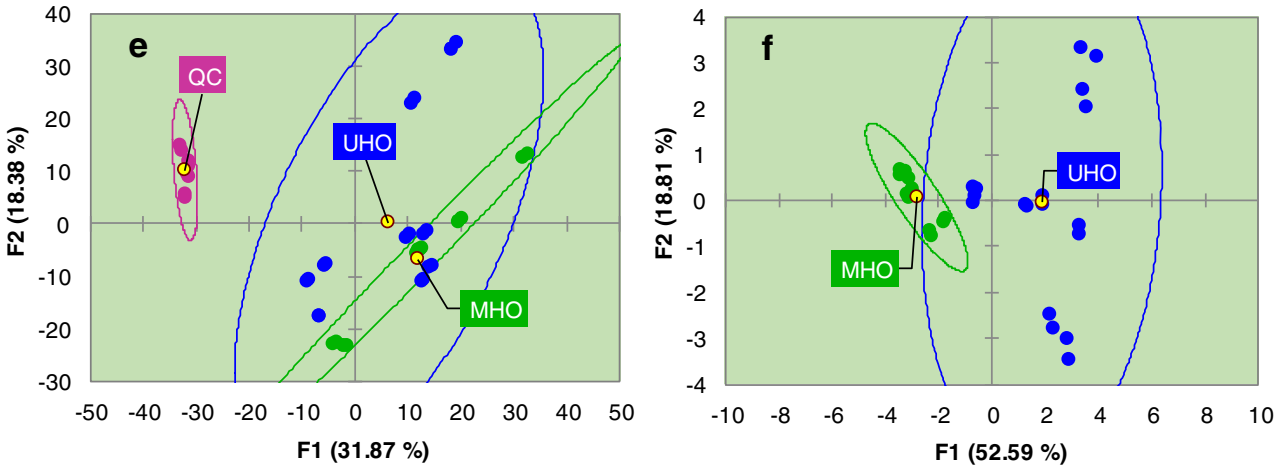

overall quality of the responses and the degree of overlap between saliva metabolome of obese patients and that of healthy individuals. Results are visualized in Fig. 3. For all detection channels (Fig. 3a, c, e), QCs are separately clustered with confidence ellipses (95\% of confidence), indicating response stability. At the same time, saliva from obese patients shows a certain degree of overlapping, in line with the common metabolic traits expected for this group. However, the saliva metabolic signature from unhealthy obese patients (UHO, blue circles) appear to be affected by a greater variability, probably due to the presence of comorbidities.

PCA score plots based on the \% response data from single (Fig. 3a and c) or fused data (Fig. 3e) are highly concordant, although with $70 \mathrm{eV}$ response data the total explained variance reached the highest value (i.e., F1 and F2, 56.3\%). The combination of results from three independent elaborations takes advantage of the complementary nature of tandem signals that enables effective cross-validation.

The next step included a feature selection by removing from the computation 2D peak regions whose variation between $\mathrm{UHO}$ and $\mathrm{MHO}$ groups was not meaningful in the description of the phenomenon. Due to the feature response distributions, which are not normal, a nonparametric test was applied, i.e., Kruskal-Wallis, with post hoc correction by Dunn's test and Bonferroni correction. Peak regions with meaningful $p$ values (alpha 0.05) were kept for further processing. 
Results are visualized in Fig. 3b d, f for $70 \mathrm{eV}, 12 \mathrm{eV}$, and fused data, respectively. The selection of variables had almost the same results in terms of retained variables (on average 100 peak regions, Table 1) but with different outcomes on the total explained variance. Fused data achieved $71 \%$ of the total variance explained by the first two components, with also an almost independent clustering of the groups of patients.

For $12 \mathrm{eV}$, the UHO samples were more dispersed, suggesting that at lower energies, the wider dynamic range of responses, although beneficial for more accurate profiling of metabolite response variations, confounds intra-class variability.

Results for fused data streams showed the highest explained variance by the first two PCs and were further explored by supervised statistics (i.e., partial least squares discriminant analysis [PLS-DA] and variable importance in projection [VIP]) to rank and select untargeted peak region features with a role in the discrimination between UHO and MUO saliva signatures and to focus identification efforts on those potential markers.

\section{Informative metabolites and their response from tandem signals}

Supervised statistics by PLS-DA and VIPs highlight discriminant peak regions within those preselected at the previous step. Features with VIPs $\geq 1$ are listed in Table 2 together with retention times in the two chromatographic dimensions $\left({ }^{1} t_{R}\right.$; $\left.{ }^{2} t_{R}\right)$, estimated linear retention index $\left(I^{T}\right)$ and literature reference (NIST library [46]), putative identification (criteria in "UT fingerprinting workflow" section), and \% of absolute response variation between samples groups (MUO vs. MHO).

Within targeted analytes, those with the larger variations were D-(+)-glucuronic acid $\gamma$-lactone (i.e., glucuronolactone $+325.2 \%)$, N-acetyl-D-glucosamine $(+128.8 \%)$, urea (+98.0\%), 2-deoxy-D-ribose (+87.8\%), N-acetylneuraminic acid methyl ester (+77.2\%), 5-aminovaleric acid (+75.3\%), 3 -methyl-2-oxobutanoic acid $(+52.7 \%)$, and sucrose $(+40.9 \%)$. All of them were upregulated in unhealthy obese patients with meaningful variations as indicated by VIP values (Table 2). On the other hand, the saliva metabolome of healthy obese individuals was connoted by a higher relative abundance of D-lactose $(+365.0 \%)$, serine $(+59.4 \%)$, and pyroglutamic acid (i.e., indicating Lproline and L-glutamine, $+17.8 \%$ ) .

Discriminant peak-region TIC responses were examined to evaluate the degree of correlation between tandem signals. Figure 4 shows linear regression results for $\mathrm{N}$-acetyl-D-glucosamine, lactose, and urea on the basis of TIC response at $12 \mathrm{eV}$ (left side) and from fused signals $(12+70 \mathrm{eV}-\mathrm{SUM}-$ right side) compared to $70 \mathrm{eV}$ taken as reference (independent variable $-x$ ). Results are satisfactory: all determination coefficients $\left(\mathrm{R}^{2}\right)$ are above 0.800 , indicating that tandem signals are highly consistent and that their combination, by fused data streams or by independent processing, might be beneficial for the dynamic range of the method.

The differential sensitivity between the two channels ( 70 and $12 \mathrm{eV}$ ) and the dissimilarity between fragmentation patterns is illustrated in Fig. 5 for one of the derivatization isomers of N-acetyl-D-glucosamine and in ESM Fig. S3 for a selection of metabolites covering different chemical classes. With image colorization, the $70 \mathrm{eV}$ channel (Fig. 5a) shows highest absolute response compared to $12 \mathrm{eV}$ (Fig. 5b), while spectra (Fig. 5c) mainly differ for the presence of lower fragments at $70 \mathrm{eV}(73,129,147 \mathrm{~m} / \mathrm{z})$ that are less represented at lower energy, in favor of larger fragments with a higher intrinsic informative role $(202,319,333 \mathrm{~m} / \mathrm{z})$.

The interindividual variations in the salivary metabolome, due to several confounding variables that might have a more decisive impact on the distribution of metabolites, can be minimized by applying data processing strategies that accumulate aligned data point features (i.e., TOF MS detector events) to generate composite class images [47] for pair-wise comparisons. Based on the fingerprinting workflow illustrated in Fig. 1 , composite class images representing the salivary metabolite signatures of healthy (MHO) and unhealthy (MUO) obese patients were built from fused data images. In this way, comparative visualization [12] was adopted to highlight pattern variations between groups. Figure 6a shows a colorized fuzzy ratio rendering [40] that illustrates the differential response between MUO (analyzed image) and MHO (reference image) composites. Areas in the pattern colored in red correspond to components whose relative TIC response (normalization is done on the total image response [40]) was higher in the reference class image (i.e., MHO), whereas areas in green are components with higher responses in the analyzed class image (i.e., MUO). Except for urea, eluting early in the chromatogram (peak region \#351) and present in higher amounts in unhealthy obese patients, there is a clear pattern of upregulated di-saccharides at higher retention indexes (Fig. 6c) that were putatively identified as lactose isomers. On the other hand, among monosaccharides (Fig. 6b), the trends for fructose (\#376) and glucose (\#27) are in contrast - see green and red colorization, respectively-while N-acetyl-Dglucosamine isomers (\#19 and \#282) were clearly upregulated in MUOs. Univariate statistics on analytes' relative distributions in sample classes, by box-plot visualization, is provided as ESM Fig. S4.

\section{Insights on saliva metabolite differential signatures}

With respect to biological interpretation of the analytical data, although sampling was limited to a few subjects within all recruited patients in the global study, it appears that saliva of metabolically unhealthy obese patients had a higher relative abundance of $\mathrm{N}$-acetyl-D-glucosamine. This amide derivative of glucose in mammals is generally bonded to serine or 


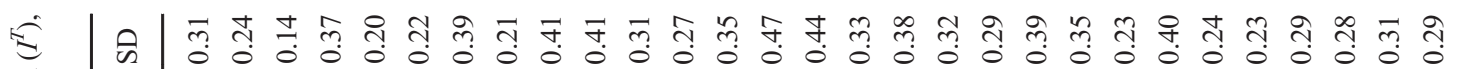

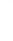

函

흥

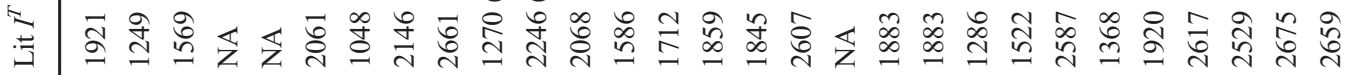

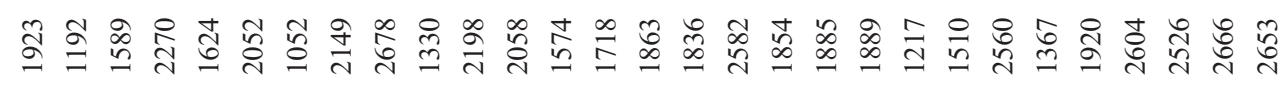
只 六

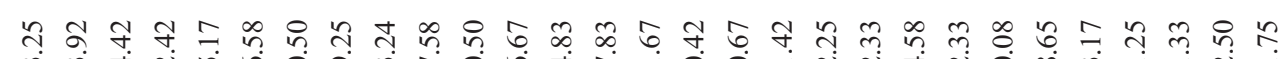

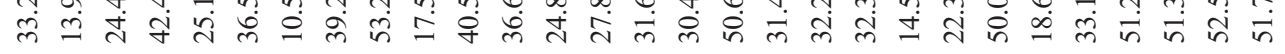
竞 
N-Acetyl-D-glucosamine 12 vs. $70 \mathrm{eV}\left(R^{2}=0.9683\right)$

N-Acetyl-D-glucosamine SUM vs. $70 \mathrm{eV}\left(\mathrm{R}^{2}=0.9812\right)$
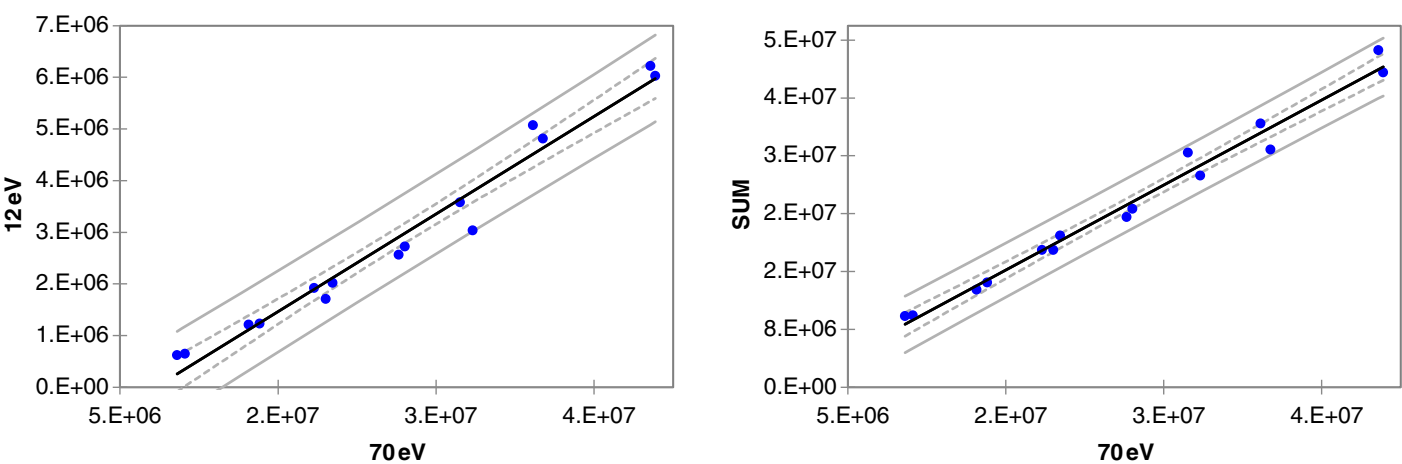

Lactose 12 vs. $70 \mathrm{eV}\left(R^{2}=0.8617\right)$

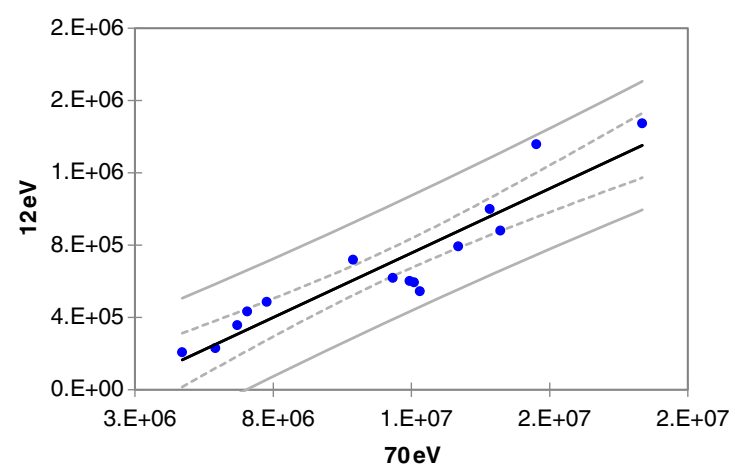

Lactose SUM vs. $70 \mathrm{eV}\left(R^{2}=0.9001\right)$

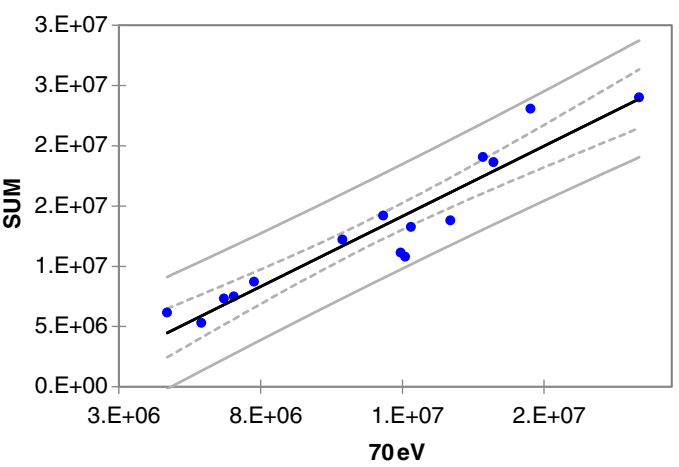

Urea 12 vs. $70 \mathrm{eV}\left(R^{2}=0.8536\right)$

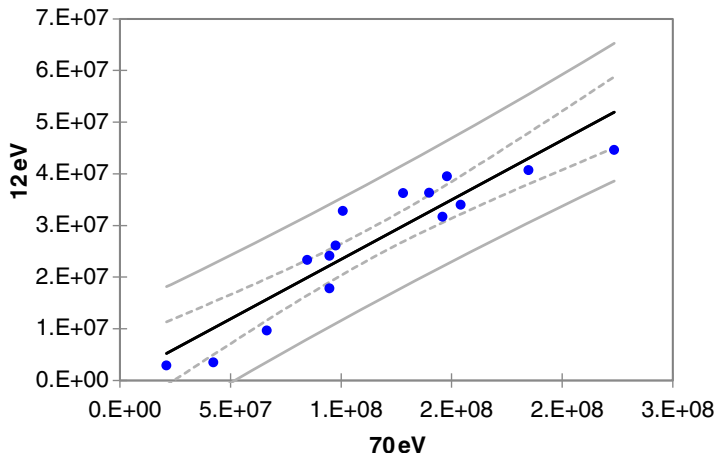

Urea SUM vs.70 eV $\left(R^{2}=0.8156\right)$

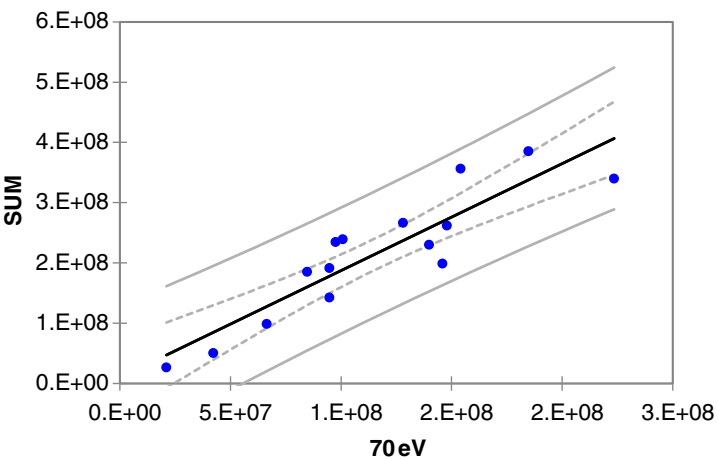

Fig. 4 Linear regression results for discriminant analytes (i.e., N-acetyl-D-glucosamine, lactose, and urea) on the basis of TIC response data at $12 \mathrm{eV}$ (left side) and from fused signals $(12+70 \mathrm{eV}-\mathrm{SUM}-$ right side) compared to $70 \mathrm{eV}$ taken as reference (independent variable $-x)$

threonine residues of a protein, giving the O-GlcNAcylation. O-GlcNAcylation appears to have a role in autophagy, insulin signaling, and stress response [48], while large amounts of OGlcNAcylation resulted in insulin resistance in adipocytes [49]. The MUO population, when profiled by multiplexed lectin microarray for carbohydrates present on proteins, resulted in clear and distinctive patterns of GlcNAcylation [28].

Urea, another informative analyte from the fingerprinting analysis, is the principal carrier of waste nitrogen derived from the protein catabolism. High levels of salivary urea were found to be abundant in chronic kidney disease patients [50], while higher D-(+)-glucuronic acid $\gamma$-lactone amounts were found in patients with hepatic steatosis [51]. On the other hand, 5 -aminovaleric acid ( $+75.3 \%$ in MUO), a lysine degradation product, is a normal metabolite present in human saliva, with a tendency to elevated concentration in patients with chronic periodontitis. Bacterial contamination and decomposition of salivary proteins is primarily responsible for elevated salivary levels of this metabolite [52].

Data on saliva level/concentration of lactose are lacking, although evidence of urine excretion of this metabolite is correlated to milk consumption [53] and, in its turn, also related to fermentation by gut microbiota that in obesity has a great impact on metabolic homeostasis [54]. 
Fig. 5 Enlarged areas of the contour plots corresponding to the elution region of a N-acetyl-Dglucosamine isomer: a $70 \mathrm{eV}$ ionization; b $12 \mathrm{eV}$ energy; c head-to-tail comparison of the resulting fragmentation pattern between the two channels
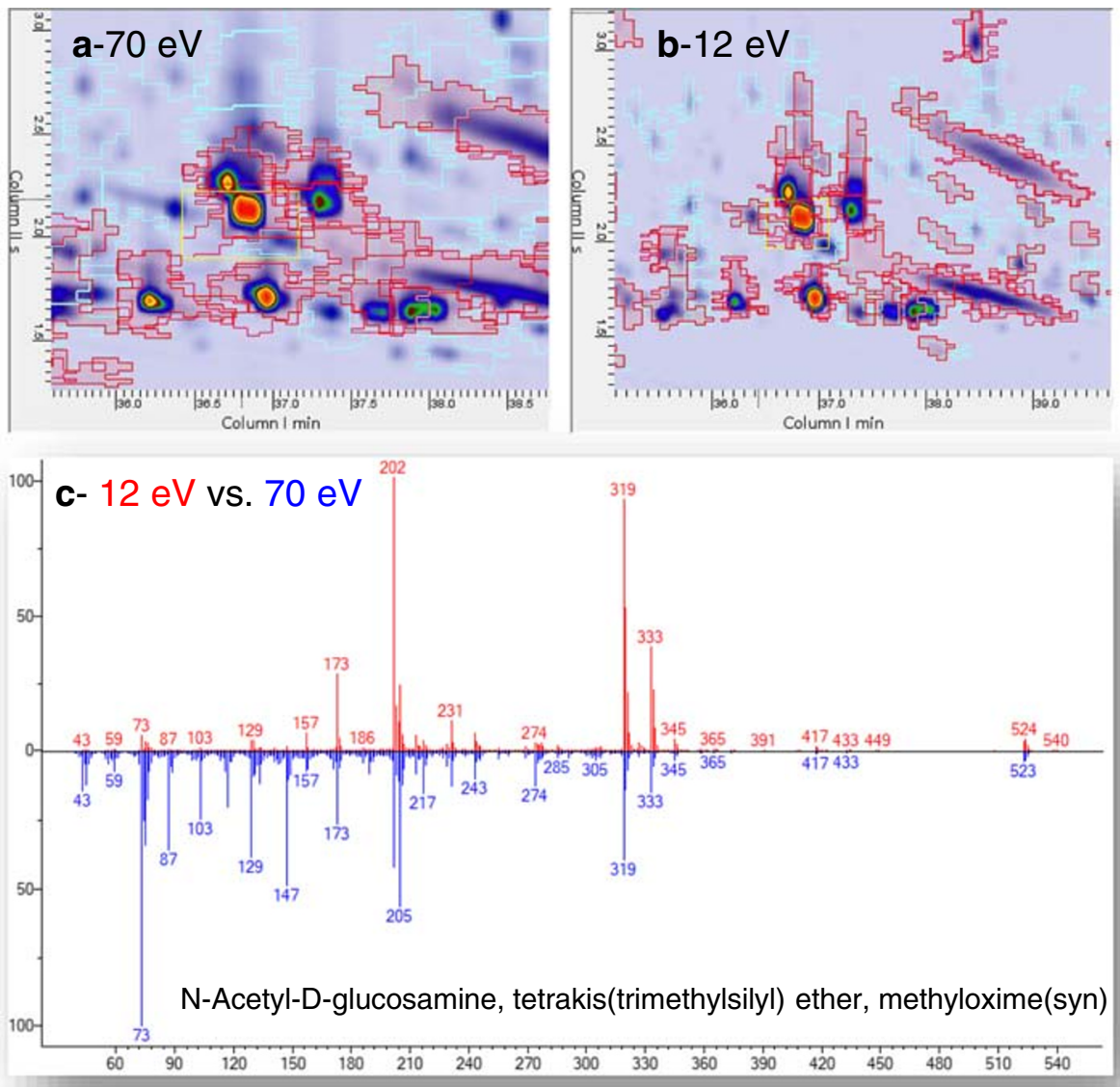
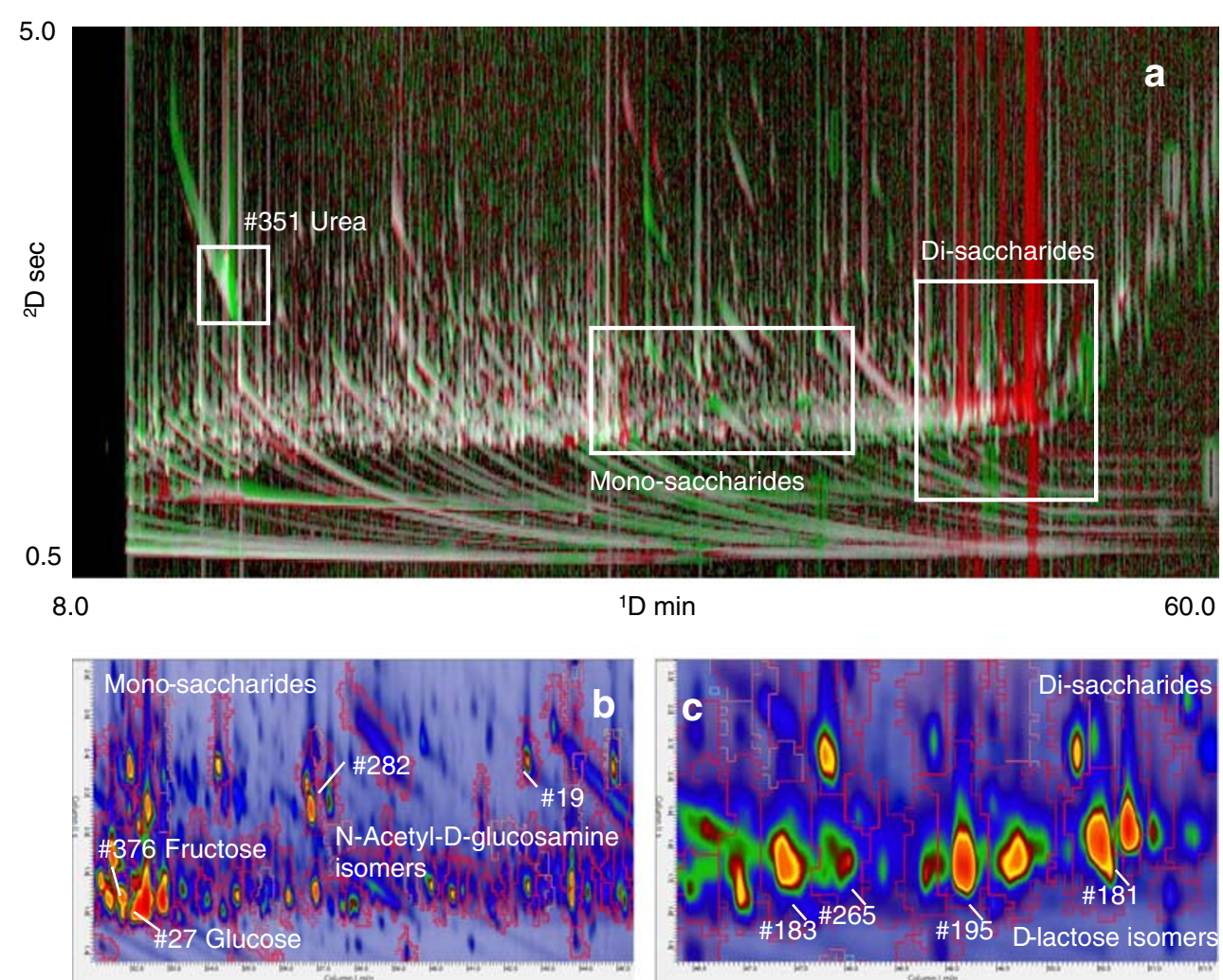

Fig. 6 Colorized fuzzy ratio rendering (a) of the differential response between composite class images from MUO (analyzed image) and $\mathrm{MHO}$ (reference image) samples. Areas in the pattern colored in red correspond to features whose relative TIC response (normalization is done on the total image response) is higher in the reference class image (i.e. MHO), while areas in green represent features with higher responses in the analyzed class image (i.e., MUO). Monosaccharide (b) and disaccharide (c) regions are detailed in enlarged areas 
When compared with QC patterns, obtained from healthy male subjects, both MHO and MUO were characterized by higher levels of butyric acid and myo-inositol, previously confirmed to be obesity markers found in blood and urine $[55,56]$.

The role of dietary carbohydrates in the development and maintenance of obesity is a topic that is receiving increasing attention [57]. Glucose is catabolized via glycolysis to pyruvate, which under aerobic conditions, is converted into acetyl coenzyme A, the entry point into the tricarboxylic acid (TCA) cycle. Under anaerobic conditions, pyruvate is instead converted into lactate by lactate dehydrogenase. In obesity, the levels of glucose, lactic acid, fructose, glycerol, mannose, sorbitol, xylose, gluconic acid, and glucuronic acid are increased [58, 59].

Overall, preliminary results from saliva signatures from obese patients indicate a hitherto unrecognized and fairly novel role for carbohydrate and amino acid metabolism in obesity while suggesting that altered metabolic fingerprints may contribute to a better understanding of the pathogenesis of different clinical contexts linked to obesity, specifically MHO vs. MUO, but also to better elucidate the inflammatory milieu detectable in the saliva of MUO, which could help to better prevent many obesity-associated pathological conditions, including cardiovascular and metabolic diseases [28].

\section{Conclusions}

Comprehensive two-dimensional gas chromatography, when combined with Tandem Ionization ${ }^{\mathrm{TM}}$ TOF MS, improves confidence in untargeted fingerprinting of complex samples. Chromatographic fingerprints from saliva metabolites obtained by summing raw MS data acquired at two ionization energies (i.e., 12 and $70 \mathrm{eV}$ ) had a larger number of reliable features (i.e., 2D peaks reliably matched across chromatograms), indicating a higher spectral consistency and stability. Low-ionization energy data showed a wider dynamic range of responses and good correlation with $70 \mathrm{eV}$ response. Moreover, lower ionization limited fragmentation by producing MS spectral signatures dominated by medium-to-high $\mathrm{m} / \mathrm{z}$ fragments with an intrinsically higher information role on analyte identity.

Although the very limited number of samples does not allow conclusive results, analytes discriminating saliva signatures of MUO vs. MHO were putatively identified. Their information role might be now be placed in the more general context of metabolic derangement caused by obesity and concurrent alterations due to comorbidities. The study provides a proof of concept on the advantages of adding extra dimensions to the analytical system adopted for chromatographic fingerprinting of biological fluids; it delineates an effective workflow that can be adopted with a larger data set.
Acknowledgements This research was funded by "ERA-NET Biomarkers for Nutrition and Health Implementing the JPI HDHL objectives" - ("SALIVAGES").

Funding Open access funding provided by Università degli Studi di Torino within the CRUI-CARE Agreement.

\section{Compliance with ethical standards}

The experimental procedure was approved by the ad hoc Ethical Research Committee of the Istituto Auxologico Italiano (Verbania, Italy). Written informed consent was obtained from the patients. The study protocol conformed to the guidelines of the European Convention on Human Rights and Biomedicine concerning biomedical research.

Conflict of interest The authors declare no conflicts of interest.

Open Access This article is licensed under a Creative Commons Attribution 4.0 International License, which permits use, sharing, adaptation, distribution and reproduction in any medium or format, as long as you give appropriate credit to the original author(s) and the source, provide a link to the Creative Commons licence, and indicate if changes were made. The images or other third party material in this article are included in the article's Creative Commons licence, unless indicated otherwise in a credit line to the material. If material is not included in the article's Creative Commons licence and your intended use is not permitted by statutory regulation or exceeds the permitted use, you will need to obtain permission directly from the copyright holder. To view a copy of this licence, visit http://creativecommons.org/licenses/by/4.0/.

\section{References}

1. Higgins Keppler EA, Jenkins CL, Davis TJ, Bean HD. Advances in the application of comprehensive two-dimensional gas chromatography in metabolomics. TrAC - Trends Anal Chem. 2018;109:27586. https://doi.org/10.1016/j.trac.2018.10.015.

2. Beccaria M, Bobak C, Maitshotlo B, Mellors TR, Purcaro G, Franchina FA, et al. Exhaled human breath analysis in active pulmonary tuberculosis diagnostics by comprehensive gas chromatography-mass spectrometry and chemometric techniques. J Breath Res. 2019;13:016005. https://doi.org/10.1088/1752-7163/ aae80e.

3. Di Giovanni N, Meuwis M-A, Louis E, Focant J-F. Untargeted serum metabolic profiling by comprehensive two-dimensional gas chromatography-high-resolution time-of-flight mass spectrometry. J Proteome Res. 2020;19:1013-28. https://doi.org/10.1021/acs. jproteome.9b00535.

4. Reichenbach SE, Tian X, Tao Q, Ledford EB, Wu Z, Fiehn O. Informatics for cross-sample analysis with comprehensive twodimensional gas chromatography and high-resolution mass spectrometry (GCxGC-HRMS). Talanta. 2011;83:1279-88. https:// doi.org/10.1016/j.talanta.2010.09.057.

5. Bressanello D, Liberto E, Collino M, Chiazza F, Mastrocola R, Reichenbach SE, et al. Combined untargeted and targeted fingerprinting by comprehensive two-dimensional gas chromatography: revealing fructose-induced changes in mice urinary metabolic signatures. Anal Bioanal Chem. 2018;410:2723-37. https://doi.org/ 10.1007/s00216-018-0950-9.

6. Pedersen HK, Gudmundsdottir V, Nielsen HB, Hyotylainen T, Nielsen T, Jensen BAH, et al. Human gut microbes impact host 
serum metabolome and insulin sensitivity. Nature. 2016;535:37681. https://doi.org/10.1038/nature18646.

7. Castillo S, Mattila I, Miettinen J, Orešič M, Hyötyläinen T. Data analysis tool for comprehensive two-dimensional gas chromatography/time-of-flight mass spectrometry. Anal Chem. 2011;83:305867. https://doi.org/10.1021/ac103308x.

8. Ros AD, Masuero D, Riccadonna S, Bubola KB, Mulinacci N, Mattivi F, et al. Complementary untargeted and targeted metabolomics for differentiation of extra virgin olive oils of different origin of purchase based on volatile and phenolic composition and sensory quality. Molecules. 2019;24:2896. https://doi.org/10.3390/ molecules24162896.

9. Purcaro G, Cordero C, Liberto E, Bicchi C, Conte LS. Toward a definition of blueprint of virgin olive oil by comprehensive twodimensional gas chromatography. J Chromatogr A. 2014;1334: 101-11. https://doi.org/10.1016/j.chroma.2014.01.067.

10. Vichi S, Pizzale L, Conte LS, Buxaderas S, Lopez-Tamames E. Solid phase microextraction in the analysis of virgin olive oil volatile fraction: characterization of virgin oils from two distinct geographical areas of northern Italy. J Agric Food Chem. 2003;51(22): 6572-7. https://doi.org/10.1021/jf030269c.

11. Cordero C, Kiefl J, Reichenbach SE, Bicchi C. Characterization of odorant patterns by comprehensive two-dimensional gas chromatography: a challenge in omic studies. TrAC - Trends Anal Chem. 2019;113:364-78. https://doi.org/10.1016/j.trac.2018.06.005.

12. Reichenbach SE, Tian X, Cordero C, Tao Q. Features for nontargeted cross-sample analysis with comprehensive twodimensional chromatography. J Chromatogr A. 2012;1226:140-8. https://doi.org/10.1016/j.chroma.2011.07.046.

13. Pedersen SD. Metabolic complications of obesity. Best Pract Res Clin Endocrinol Metab. 2013;27:179-93. https://doi.org/10.1016/j. beem.2013.02.004.

14. Reichenbach SE, Carr PW, Stoll DR, Tao Q. Smart templates for peak pattern matching with comprehensive two-dimensional liquid chromatography. J Chromatogr A. 2009;1216:3458-66. https://doi. org/10.1016/j.chroma.2008.09.058.

15. Beccaria M, Franchina FA, Nasir M, Mellors T, Hill JE, Purcaro G. Investigation of mycobacteria fatty acid profile using different ionization energies in GC-MS. Anal Bioanal Chem. 2018;410:798796. https://doi.org/10.1007/s00216-018-1421-z.

16. Markes International. Select-eV: the next generation of ion source technology. Tech Note Application N 528. 2016.

17. Cordero C, Guglielmetti A, Bicchi C, Liberto E, Baroux L, Merle P, et al. Comprehensive two-dimensional gas chromatography coupled with time of flight mass spectrometry featuring tandem ionization: challenges and opportunities for accurate fingerprinting studies. J Chromatogr A. 2019;1597:132-41. https://doi.org/10. 1016/j.chroma.2019.03.025.

18. Alam MS, Stark C, Harrison RM. Using variable ionization energy time-of-flight mass spectrometry with comprehensive GC??GC to identify isomeric species. Anal Chem. 2016;88:4211-20. https:// doi.org/10.1021/acs.analchem.5b03122.

19. Freye CE, Moore NR, Synovec RE. Enhancing the chemical selectivity in discovery-based analysis with tandem ionization time-offlight mass spectrometry detection for comprehensive twodimensional gas chromatography. J Chromatogr A. 2018;1537: 99-108. https://doi.org/10.1016/j.chroma.2018.01.008.

20. Dubois LM, Perrault KA, Stefanuto PH, Koschinski S, Edwards M, $\mathrm{McGregor} \mathrm{L,} \mathrm{et} \mathrm{al.} \mathrm{Thermal} \mathrm{desorption} \mathrm{comprehensive} \mathrm{two-}$ dimensional gas chromatography coupled to variable-energy electron ionization time-of-flight mass spectrometry for monitoring subtle changes in volatile organic compound profiles of human blood. J Chromatogr A. 2017;1501:117-27. https://doi.org/10. 1016/j.chroma.2017.04.026.

21. Cialiè Rosso M, Mazzucotelli M, Bicchi C, Charron M, Manini F, Menta R, et al. Adding extra-dimensions to hazelnuts primary metabolome fingerprinting by comprehensive two-dimensional gas chromatography combined with time-of-flight mass spectrometry featuring tandem ionization: insights on the aroma potential. J Chromatogr A. 1614;2020:1-11. https://doi.org/10.1016/j.chroma. 2019.460739.

22. Wong DTW. Salivaomics. J Am Dent Assoc. 2012;143:19-24. https://doi.org/10.14219/jada.archive.2012.0339.

23. Yoshizawa JM, Schafer CA, Schafer JJ, Farrell JJ, Paster BJ, Wong DTW. Salivary biomarkers: toward future clinical and diagnostic utilities. Clin Microbiol Rev. 2013;26:781-91. https://doi.org/10. 1128/CMR.00021-13.

24. Choromańska K, Choromańska B, Dąbrowska E, Bączek W, Myśliwiec P, Dadan J, et al. Saliva of obese patients - is it different? Postepy Hig Med Dosw. 2015;69:1190-5. https://doi.org/10.5604/ 17322693.1176778.

25. Lehmann-Kalata A, Miechowicz I, Korybalska K, Swora-Cwynar E, Czepulis N, Łuczak J, et al. Salivary fingerprint of simple obesity. Cytokine. 2018;110:174-80. https://doi.org/10.1016/j.cyto. 2018.05.006.

26. Knaś M, Maciejczyk M, Sawicka K, Hady HR, Niczyporuk M, Ładny JR, et al. Impact of morbid obesity and bariatric surgery on antioxidant/oxidant balance of the unstimulated and stimulated human saliva. J Oral Pathol Med. 2016;45:455-64. https://doi.org/10. 1111/jop.12383.

27. Blüher M. Metabolically healthy obesity. Endocr Rev. 2020;41: 405-20. https://doi.org/10.1210/endrev/bnaa004.

28. Collotta D, Cordero C, Gerlach JQ, Liberto E, Chiazza F, Cialiè Rosso M, et al. Pilot study on comparative profiling of biofluids (plasma, urine and saliva) from metabolically healthy and metabolically unhealthy obese subject. In: Proceedings of the $39^{\circ}$ Congresso Nazionale della Società Italiana di Farmacologia Firenze, 20-23 Novembre, 2019. 2019.

29. Mai S, Grugni G, Mele C, Vietti R, Vigna L, Sartorio A, et al. Irisin levels in genetic and essential obesity: clues for a potential dual role. Sci Rep. 2020;10:1-9. https://doi.org/10.1038/s41598-020-578555.

30. Grundy SM, Cleeman JI, Daniels SR, Donato KA, Eckel RH, Franklin BA, et al. Diagnosis and management of the metabolic syndrome: an American Heart Association/National Heart, Lung, and Blood Institute scientific statement. Curr Opin Cardiol. 2006;21:1-6. https://doi.org/10.1097/01.hco.0000200416.65370. a0.

31. Zhang Q, Wang G, Du Y, Zhu L, Jiye A. GC/MS analysis of the rat urine for metabonomic research. J Chromatogr B. 2007;854:20-5. https://doi.org/10.1016/j.jchromb.2007.03.048.

32. Eurachem. Eurachem Guide: The Fitness for Purpose of Analytical Methods - A Laboratory Guide to Method Validation and Related Topics. 2014.

33. Magagna F, Valverde-Som L, Ruíz-Samblás C, CuadrosRodríguez L, Reichenbach SE, Bicchi C, et al. Combined untargeted and targeted fingerprinting with comprehensive twodimensional chromatography for volatiles and ripening indicators in olive oil. Anal Chim Acta. 2016;936:245-58. https://doi.org/10. 1016/j.aca.2016.07.005.

34. Reichenbach SE, Zini CA, Nicolli KP, Welke JE, Cordero C, Tao Q. Benchmarking machine learning methods for comprehensive chemical fingerprinting and pattern recognition. J Chromatogr A. 2019;1595:158-67. https://doi.org/10.1016/j.chroma.2019.02.027.

35. Stilo F, Liberto E, Reichenbach SE, Tao Q, Bicchi C, Cordero C. Untargeted and targeted fingerprinting of extra virgin olive oil volatiles by comprehensive two-dimensional gas chromatography with mass spectrometry: challenges in long-term studies. J Agric Food Chem. 2019;67:5289-302. https://doi.org/10.1021/acs.jafc. $9 \mathrm{~b} 01661$.

36. Nicolotti L, Cordero C, Bressanello D, Cagliero C, Liberto E, Magagna F, et al. Parallel dual secondary column-dual detection: 
a further way of enhancing the informative potential of twodimensional comprehensive gas chromatography. J Chromatogr A. 2014;1360:264-74. https://doi.org/10.1016/j.chroma.2014.07. 081.

37. Sgorbini B, Cagliero C, Boggia L, Liberto E, Reichenbach SE, Rubiolo P, et al. Parallel dual secondary-column-dual detection comprehensive two-dimensional gas chromatography: a flexible and reliable analytical tool for essential oils quantitative profiling. Flavour Fragr J. 2015;30:366-80. https://doi.org/10.1002/ffj.3255.

38. Reichenbach SE, Rempe DW, Tao Q, Bressanello D, Liberto E, Bicchi C, et al. Alignment for comprehensive two-dimensional gas chromatography with dual secondary columns and detectors. Anal Chem. 2015;87:10056-63. https://doi.org/10.1021/acs.analchem. $5 \mathrm{~b} 02718$.

39. Reichenbach SE, Tian X, Boateng AA, Mullen CA, Cordero C, Tao Q. Reliable peak selection for multisample analysis with comprehensive two-dimensional chromatography. Anal Chem. 2013;85: 4974-81. https://doi.org/10.1021/ac303773v.

40. Bressanello D, Liberto E, Collino M, Reichenbach SE, Benetti E, Chiazza F, et al. Urinary metabolic fingerprinting of mice with dietinduced metabolic derangements by parallel dual secondary column-dual detection two-dimensional comprehensive gas chromatography. J Chromatogr A. 2014;1361:265-76. https://doi.org/ 10.1016/j.chroma.2014.08.015.

41. Dame ZT, Aziat F, Mandal R, Krishnamurthy R, Bouatra S, Borzouie $\mathrm{S}$, et al. The human saliva metabolome. Metabolomics. 2015;11:1864-83. https://doi.org/10.1007/s11306-015-0840-5.

42. Stilo F, Tredici G, Bicchi C, Robbat A, Morimoto J, Cordero C. Climate and processing effects on tea (Camellia sinensis L. Kuntze) metabolome: accurate profiling and fingerprinting by comprehensive two-dimensional gas chromatography/time-of-flight mass spectrometry. Molecules. 2020;25:2447. https://doi.org/10.3390/ molecules 25102447

43. Reichenbach SE, Shi J. Two-Dimensional Cubic Convolution for One-Pass Image Restoration and Reconstruction. IGARSS 2004. 2004 IEEE International Geoscience and Remote Sensing Symposium, Anchorage, AK, 2004, pp. 2074-206a vol. 3 . 2004. https://doi.org/10.1109/IGARSS.2004.1370759.

44. Sternberg. Biomedical Image Processing. Computer. 1983;16:2234. https://doi.org/10.1109/MC.1983.1654163.

45. Scientific N, Databases T. NIST/EPA/NIH mass spectral library with search program: (data version: NIST 08, Software Version 2.0f). 2010. 2005 .

46. Tret'yakov KV. Retention Data. NIST Mass Spectrometry Data Center. Gaithersburg, MD 20899-8362. 2007.

47. Stilo F, Gabetti E, Spigolon N, Genova G, Somenzi M, Fontana M, et al. Chromatographic fingerprinting of hazelnuts volatiles by comprehensive two-dimensional gas chromatography coupled with time of flight mass spectrometry: challenges in defining odorant patterns related to sensory defects. In: Armstrong D, Schug KA, editors. Proceedings of the 43rd international symposium on capillary chromatography \& the 16 th GCxGC symposium. Texas, USA: Fort Worth; 2019.

48. Levine ZG, Walker S. The biochemistry of O-GlcNAc transferase : which functions make it essential in mammalian cells ? Annu Rev Biochem. 2016;85:631-57. https://doi.org/10.1146/annurevbiochem-060713-035344.

49. Vosseller K, Wells L, Lane MD, Hart GW. Elevated nucleocytoplasmic glycosylation by O-GlcNAc results in insulin resistance associated with defects in Akt activation in 3T3-L1 adipocytes. PNAS. 2002;99:5313-8. https://doi.org/10.1073/pnas. 072072399

50. Renda R. Can salivary creatinine and urea levels be used to diagnose chronic kidney disease in children as accurately as serum creatinine and urea levels ? A case - control study. Ren Fail. 2017;0:452-7. https://doi.org/10.1080/0886022X.2017.1308256.

51. Troisi J, Belmonte F, Bisogno A, Pierri L, Colucci A, Scala G, et al. Metabolomic salivary signature of pediatric obesity related liver disease and metabolic syndrome. Nutrients. 2019;11:274. https:// doi.org/10.3390/nu11020274.

52. Syrjänen S, Piironen P, Markkanen H. Free amino-acid content of wax-stimulated human whole saliva as related to periodontal disease. Arch Oral Biol. 1987;32:607-10. https://doi.org/10.1016/ 0003-9969(87)90032-X.

53. Beauchamp GK, Mennella JA. Early flavor learning and its impact on later feeding behavior. J Pediatr Gastroenterol Nutr. 2009;48: 25-30. https://doi.org/10.1097/MPG.0b013e31819774a5.

54. Muscogiuri G, Cantone E, Cassarano S, Tuccinardi D, Barrea L, Savastano S, et al. Gut microbiota: a new path to treat obesity. Int J Obes Suppl. 2019;9:10-9. https://doi.org/10.1038/s41367-0190011-7.

55. Kasubuchi M, Hasegawa S, Hiramatsu T, Ichimura A, Kimura I. Dietary gut microbial metabolites, short-chain fatty acids, and Host Metabolic Regulation. Nutrients. 2015;14:2839-49. https://doi.org/ 10.3390/nu7042839.

56. Hwa J, Won H, Eun Y, Hee J, Soon K, Jin H. Urinary chiro- and myo-inositol levels as a biological marker for type 2 diabetes mellitus. Dis Markers. 2012;33:193-9. https://doi.org/10.3233/ DMA-2012-0925.

57. Siri-Tarino PW, Sun Q, Hu FB, Krauss RM. Saturated fat, carbohydrate, and cardiovascular disease. Am J Clin Nutr. 2010;91:5029. https://doi.org/10.3945/ajcn.2008.26285.

58. Moore SC, Matthews CE, Sampson JN, Stolzenberg-Solomon RZ, Zheng W, Cai Q, et al. Human metabolic correlates of body mass index. Metabolomics. 2014;10:259-69. https://doi.org/10.1007/ s11306-013-0574-1.

59. Rauschert S, Uhl O, Koletzko B, Hellmuth C. Metabolomic biomarkers for obesity in humans: a short review. Ann Nutr Metab. 2014;64:314-24. https://doi.org/10.1159/000365040.

Publisher's note Springer Nature remains neutral with regard to jurisdictional claims in published maps and institutional affiliations. 